Número 4, Septiembre 2015. No 04/02. ISSN: 2014-5993

http://dx.doi.org/10.5565/rev/grafowp. 15

\title{
La enfermedad de Alzheimer desde el análisis de redes sociales
}

Nombre: Jaime Llopis Cañameras

Afiliación: Parc Sanitari Sant Joan de Déu - Hospital General (Sant Boi de Llobregat Barcelona).

Dirección electrónica: jaimellop@gmail.com

\section{Resumen}

El estudio de las redes personales de 23 cuidadores/as de personas afectadas por la enfermedad de Alzheimer pone de manifiesto los profundos cambios que se producen en el entorno social de estas personas. En general las redes se hacen más pequeñas y se concentran en las funciones de apoyo, siguiendo varias evoluciones posibles que hemos caracterizado según una tipología: redes reducidas, institucionalizadas, medicalizadas y normalizadas. Esta tipología puede depender del estado de evolución de la enfermedad.

Palabras clave: Alzheimer, redes personales, apoyo social.

\section{Abstract}

The study of the personal networks of 23 caregivers / as of people affected by Alzheimer's disease reveals the profound changes occurring in the social environment of these people. Overall networks become smaller and focus on support functions, following several possible developments that have characterized as a typology: small, institutionalized and standardized networks medicalized. This classification may depend on the state of evolution of the disease.

Keywords: Alzheimer, personal networks, social support.

\section{Introducción}

Uno de los efectos de los constantes avances experimentado en el ámbito de la medicina y la institucionalización de la salud ha sido el aumento generalizado de la esperanza de vida. Esta circunstancia ha provocado un envejecimiento de la población y como consecuencia un considerable aumento de las enfermedades crónicas y degenerativas (Bazo, 1998; Isla, 2000).

La Organización Mundial de la Salud - OMS, viene alertando del incremento de las enfermedades neurodegenerativas (OMS, 2007), con 24 millones de personas padeciendo 
Número 4, Septiembre 2015. No 04/02. ISSN: 2014-5993

http://dx.doi.org/10.5565/rev/grafowp. 15

Alzheimer y otras demencias. Por otra parte, el Informe Mundial sobre el Alzheimer publicado por Alzheimer Disease International (2009) pone de manifiesto que cada veinte años se duplicará esta cifra, con una previsión para el 2030 de 65,7 millones de pacientes y 115,4 millones para el 2050.

Pero ¿qué es el Alzheimer? La Enfermedad de Alzheimer (a partir de ahora EA), es una enfermedad neurodegenerativa de carácter progresiva e irreversible. Progresivamente se van perdiendo células cerebrales que provocan un deterioro de la memoria, aptitudes del pensamiento y alteraciones en la conducta.

El diagnóstico de la EA en un paciente se centra básicamente en la propia historia clínica, el estudio neuro-psicológico y las pruebas complementarias como el test de Minimental. Según los especialistas las causas provienen de determinados factores de riesgo demográficos como son: la edad, el género y antecedentes médicos como la Diabetes Mellitus, el tabaquismo, la Hipertensión Arterial o la colesterinemia. No se descartan los factores genéticos, aunque son pocos los casos que se presentan por tales motivos (Farreras \& Rozman, 1985; Callen; 2014). El tratamiento actual se dirige a tratar los síntomas que sin duda mejoran la calidad de la vida diaria del paciente.

Por otra parte en el ámbito social los modelos familiares están sufriendo cambios importantes: disminución del número de miembros, constitución de familias monoparentales, incorporación de la mujer al mercado laboral, etc. Estos cambios en los modelos tradicionales en la familia, ponen en peligro la continuidad de los cuidados que se han producido hasta el momento (Bazo, 1998). Los gestores sanitarios observan como esta nueva situación pone en peligro los sistemas de salud nacionales, cada vez peor financiados. El progresivo envejecimiento de la población comporta además un mayor uso de los servicios sanitarios y en consecuencia un incremento del gasto total.

Por todo ello la sociedad en general y la Administración en particular están adoptando nuevas políticas en el ámbito de los cuidados "informales" para complementar un Estado del bienestar incapaz de cubrir las necesidades de la población.

Pero para hablar de "cuidados" es necesario definir dicho término. El acto de cuidar es inherente al ser humano y ha sido tradicionalmente relegado a una actividad doméstica propia de la mujer (De la Cuesta, 2004). Como señalaba Virginia Henderson (Callen, 2014):

"Cuidar es asistir al individuo enfermo en la realización de aquellas actividades que promuevan la salud o su recuperación, que él llevaría a cabo si tuviera la fuerza, la 
Número 4, Septiembre 2015. No 04/02. ISSN: 2014-5993

http://dx.doi.org/10.5565/rev/grafowp.15

voluntad o el conocimiento necesario, y hacerlo de tal manera que le ayude a recobrar su independencia con la mayor brevedad posible".

A su vez es necesario distinguir el cuidado "formal" o profesional de aquel que se realiza generalmente en el núcleo familiar dirigida a la atención de un familiar dependiente y llevado a cabo por familiares o amigos, los cuales no perciben remuneración alguna y generalmente sin conocimientos previos, Además su dedicación no tiene horarios (Isla, 2000; Aldana y Guarino, 2012).

Siguiendo a Aldana y Guarino, es necesario distinguir además al "cuidador principal" del "cuidador secundario" y del "cuidador ausente". El "cuidador principal" es aquella persona que asume la responsabilidad de tales cuidados acompañándole la mayor parte del tiempo. El "cuidador secundario" el aquella persona que ayuda al cuidador principal cuando éste lo precisa. Finalmente el "cuidador ausente" es aquella persona que forma parte del entorno más cercano del paciente pero que no colabora en los cuidados a pesar de que por razones familiares o afectivas así se espera de él.

La mayoría de los autores señalan que la base principal de los cuidados que recibe un enfermo radica en la propia familia (Úbeda y Roca, 2008; Molina, Lañez y Lañez, 2005). La familia adquiere un rol fundamental ante los cuidados de un familiar enfermo de forma irrenunciable e ineludible. Estos cuidados generalmente recaen en la mujer que en muchas ocasiones pasan de forma desapercibida y en consecuencia sin un debido reconocimiento social. Esta dedicación por parte de la mujer en numerosas ocasiones conlleva al abandono del mercado laboral de forma temporal o definitiva (Toribio et alt., 2012).

Merece especial atención además el elevado coste que supone el cuidado de un familiar enfermo. Según un estudio realizado por la Fundación Española de Enfermedades Neurológicas (Prieto et. al. 2011), el gasto de los cuidados aumenta a medida que evoluciona la enfermedad. La familia asume el $87 \%$ del coste total de tales gastos agravándose en los casos de institucionalización del enfermo.

Como consecuencia del aumento de las enfermedades neurodegenerativas y de la importancia adquirida de los cuidados informales, entre otros aspectos, en las últimas décadas han adquirido una notable importancia los estudios en los que se relaciona el Apoyo Social y la Salud. Cuatro son las líneas de investigación que se vienen realizando desde mediados del pasado siglo XX (De la Cuesta, 2004): (a) estudios sobre la carga del cuidador y elaboración de instrumentos de medida a tal efecto (b) estudios dirigidos a examinar los procesos de los cuidados (c) estudios que ponen en evidencia las vivencias del cuidar a 
Número 4, Septiembre 2015. No 04/02. ISSN: 2014-5993

http://dx.doi.org/10.5565/rev/grafowp. 15

otros y (d) estudios que evalúan la efectividad de las intervenciones para disminuir la sobrecarga de los cuidadores.

Al mismo tiempo, la importancia de los estudios de las redes sociales en el ámbito de la salud se ha puesto de manifiesto más recientemente (Maya, et. al. 1999; Hollstein, 2002; Llopis 2006 y 2011; Zúñiga, 2007; entre otros).

La investigación que a continuación presentamos precisamente tiene como objetivo principal el estudio de las redes personales en el ámbito de la salud.

\section{Objetivos y metodología}

La investigación que aquí se presenta ha sido de carácter no experimental, transversal y mixta (cualitativa y cuantitativa). Al mismo tiempo no se ha perseguido una significación estadística de los resultados obtenidos, sino por el contrario la obtención del mayor número de casos distintos que pongan de manifiesto la mayor diversidad posible. Ha tenido una duración de 21 meses y ha contado con una beca de investigación otorgada por el Parc Sanitari de Sant Joan de Déu.

Esta investigación fue previamente aprobada por el Comité de Ética y por el Comité de Recerca del Parc Sanitari de Sant Joan de Déu. En todo momento se han seguido los principios éticos de investigación, entregándose el "consentimiento Informado y la hoja informativa" de la investigación a cada una de las personas participantes.

El objetivo principal de esta investigación se ha dirigido a determinar si las Redes Personales (a partir de ahora RP) de las personas que cuidan a familiares que padecen Alzheimer se ven modificadas como consecuencia de esa nueva situación estresante.

Por otra parte los objetivos específicos se han dirigido en primer lugar al análisis de las RP de tales cuidadores/as. En segundo lugar el análisis de los posibles cambios sufridos como consecuencia de dicha enfermedad de su familiar y finalmente, el análisis del tipo de apoyo que tales cuidadores/as solicitan, así como la procedencia del mismo.

Para la realización de esta investigación se ha tomado como participantes a los/las cuidadores/as de familiares con la enfermedad de Alzheimer y residentes en el área de influencia del Hospital General del Parc Sanitari de Sant Joan de Déu ${ }^{1}$. El número de

\footnotetext{
1 El hospital General forma parte de complejo asistencial del Parc Sanitari de Sant Joan de Déu en Sant Boi de Llobregat, formado por el antiguo Hospital Psiquiátrico de Sant Joan de Déu y el Hospital General. Cuenta con especialidades médicas y quirúrgicas, así como con unidades docentes y de investigación.
} 


\section{GRAFO Working Papers}

Número 4, Septiembre 2015. No 04/02. ISSN: 2014-5993

http://dx.doi.org/10.5565/rev/grafowp. 15

participantes en esta investigación se han distribuido en cuatro perfiles obtenidos de la práctica diaria, siendo el total de casos obtenidos de 23 (Ver tabla I).

\begin{tabular}{|l|c|}
\hline \multicolumn{1}{|c|}{ GRUPO } & N \\
\hline Hombre cuidador de sus padres & 4 \\
\hline Hombre cuidador de su pareja & 3 \\
\hline Mujer cuidadora de sus padres & 10 \\
\hline Mujer cuidadora de su pareja & 6 \\
\hline TOTAL & 23 \\
\hline
\end{tabular}

Tabla I: Distribución de los/las participantes en la investigación.

El total de casos a analizar al inicio de la investigación se establecieron en 40 , distribuidos de manera uniforme entre los cuatro grupos señalados. La dificultad en contactar con varones cuidadores tanto de sus padres como de su pareja, así como con mujeres cuidadoras de sus parejas, explica el menor número de casos.

Los criterios de inclusión de los/las participantes en nuestra investigación han sido los siguientes: (1) Cuidadores de pacientes con diagnóstico de demencia según los criterios DSM-IV-TR y los criterios INCDS-ADRDA; (2) Disponibilidad para realizar el estudio; (3) Cuidadores que hayan realizado su consentimiento por escrito para participar en la investigación; (4) Cuidadores mayores de 18 años y (5) Que dispongan de tiempo necesario para realizar las técnicas previstas.

La selección de participantes se realizado de forma consecutiva hasta completar el número deseado, entre las personas asistentes a las sesiones programadas por la Escola de Cuidadors del Parc Sanitari y por aquellas personas que durante la investigación acudieron a la Consulta Médica de Neurología del Hospital General.

También es importante señalar que en esta investigación ha participado Antoni Callen Médico Adjunto del Servicio de Neurología y Director de la Escola de Cuidadors del Parc Sanitari. 
Número 4, Septiembre 2015. No 04/02. ISSN: 2014-5993

http://dx.doi.org/10.5565/rev/grafowp. 15

\section{Técnicas}

Tres han sido las técnicas empleadas en esta investigación: (1) La observación (2) el Cuestionario Zarit y (3) El Análisis de Redes Personales.

La observación

Durante la investigación el investigador principal ha asistido a un total de cinco sesiones organizadas por la Escola de Cuidadors del Parc Sanitari. Esta Escuela fue creada en el año 2008 como consecuencia de la necesidad de facilitar la labor diaria de las/los cuidadoras/es.

Entre sus objetivos debemos destacar la ampliación de información que se ofrece y se favorece a evitar el agotamiento de tales cuidadoras/es que supone el cuidado diario de un familiar que padece la Enfermedad de Alzheimer. Cuenta con un equipo multidisciplinar que implica a servicios y especialidades como son: Neurología, Psiquiatría, Geriatría, Enfermería, Nutrición y Dietética, Trabajo Social, Atención Espiritual y Antropología.

Para ello la Escola de Cuidadors organiza en el trascurso del año distintas sesiones dirigidas a cuidadoras/es aportando información y facilitando la comunicación de sus angustias y temores entre iguales. Durante las sesiones de dicha Escola se han podido observar tanto las actitudes de las/los cuidadoras/es que atienden a sus familiares, como sus emociones y angustias. De esta manera los resultados obtenidos tienen un valor especial que con otras técnicas serían difíciles de obtener.

El cuestionario Zarit

El Test sobre la carga del cuidador (Zarit, 1980)) cuantifica el grado de sobrecarga del Cuidador/a. Está formado por 22 preguntas cerradas en la que la persona participante debe responder con una puntuación graduada de (0: Nunca) a (4: Casi siempre). Ha sido distribuido antes de la sesión de análisis de redes por el propio investigador o en la consulta de Neurología.

\section{Análisis de Redes Personales}

Para dicho análisis se ha utilizado el software EgoNet (http: sourceforge.net/projects/egonet). Dicho programa nos permite la visualización entre otros, de aspectos estructurales de las redes de los/las participantes, así como los atributos de las personas citadas por cada 


\section{GRAFO Working Papers}

Número 4, Septiembre 2015. No 04/02. ISSN: 2014-5993

http://dx.doi.org/10.5565/rev/grafowp. 15

participante. Consta de cuatro módulos interrelacionados cuyas preguntas - variables - han sido previamente introducidas por el investigador. El primer módulo nos presenta los atributos de la persona participante (A partir de ahora citaremos como ego). El segundo módulo nos presenta un generador de nombres. Ego debe citar un número determinado de personas con los que se relaciona de forma habitual (A partir de ahora citaremos como alter/alteri). Para esta investigación se estableció como máximo 23 personas citadas por cada ego. A continuación el tercer módulo nos presenta los atributos de cada uno de los alteri citados por ego. Finalmente en el cuarto módulo se nos pregunta por la relación existente entre sí de cada uno de los alteri citados. Una vez finalizada la recogida de datos de cada una de las personas participantes, se procede a la visualización de la red en cuestión. En ella a través de las formas, tamaño y colores podremos observar la red personal de cada participante, así como obtener datos numéricos que posteriormente se podrán analizar a través de programas estadísticos.

\section{Resultados}

Los resultados obtenidos proceden de la triangulación de los datos arrojados por cada una de las técnicas empleadas. Por dicha causa haremos especial énfasis a los resultados obtenidos a través de la observación en las distintas sesiones de la Escola Cuidadors, así como del propio análisis de las RP de las personas que han participado.

\section{Procedentes de la observación.}

Durante las sesiones en las que el investigador ha estado presente, han sido numerosas las manifestaciones de sufrimiento, angustia y verbalización tanto de sentimientos como de temores expresadas por las/los cuidadoras/es. Varios han sido los aspectos que constantemente han ido surgiendo y que a continuación presentamos.

- La evolución del proceso de duelo - en términos de Kübler-Ross (Kübler-Ross; 1993; Tizón, 2004). Las cuidadoras se sienten culpables por una asistencia inadecuada del familiar con la enfermedad. También se sienten culpables de la institucionalización de dicho familiar enfermo en un centro ante la dificultad que encierra la asistencia en las fases más avanzadas de la EA. A estos sentimientos se les une fases de depresión, miedo a la evolución tanto de la enfermedad como de las reacciones del enfermo. Tampoco podemos olvidar las constantes somatizaciones de enfermedades como consecuencia del estrés soportado. 
Número 4, Septiembre 2015. No 04/02. ISSN: 2014-5993

http://dx.doi.org/10.5565/rev/grafowp. 15

- Cambios en la relación de pareja. En numerosas ocasiones se hace mención a la pérdida de las relaciones entre cuidador/a y su pareja. Frases como la existencia de una "renuncia sexual" o "me doy cuenta que la estoy perdiendo" son frecuentes.

- Cambios en la vivienda. En numerosas ocasiones la pérdida de autonomía por parte de la persona afectada por la enfermedad, obliga a un replanteamiento del núcleo familiar. Las hijas - con mayor frecuencia - se ven obligadas por connotaciones espirituales o por la feminización de los cuidados hacia el enfermo, a acoger a sus progenitores en su domicilio. Esta circunstancia obliga en muchas ocasiones a adecuar el espacio para acoger al nuevo miembro, con la pérdida de intimidad que supone para tales cuidadoras/es.

- Modificación de sus redes sociales. Tanto cuidadoras como cuidadores son conscientes que la dedicación casi exclusiva de los cuidados a la persona enferma conlleva a una pérdida de las relaciones sociales. Señalan que muchos amigos se pierden como consecuencia del aislamiento social que poco a poco se va originando. Este aspecto podremos observarlo con mayor detenimiento al analizar las redes personales de las personas que han participado en nuestra investigación.

- Los cuidadoras/es señalan que las principales fuentes de apoyo proceden de su pareja y de sus hijos/as. Este aspecto también lo observaremos más adelante.

- Finalmente cabe señalar la mayor asistencia de mujeres a las distintas sesiones convocadas por la Escola de Cuidadors.

\section{Procedentes del Análisis de Redes Personales}

Mediante el análisis de las RP de las/los participantes se han podido analizar tres aspectos fundamentales: (1) Las propias Redes Personales (2) La obtención de un perfil de la persona cuidadora (3) Quién atiende al cuidador.

En nuestra investigación se han obtenido cuatro tipos de redes personales. En primer lugar aquellas redes de estructura reducida. Esta disminución de la red genera un aislamiento social como consecuencia de la sobrecarga del cuidador. En segundo lugar aquellas redes 


\section{GRAFO Working Papers}

Número 4, Septiembre 2015. No 04/02. ISSN: 2014-5993

http://dx.doi.org/10.5565/rev/grafowp. 15

que podemos denominar institucionalizadas. A tales redes se incorporan a otros cuidadores/as a través de una Asociación o Entidad. En tercer lugar las redes que podemos denominar medicalizadas, entendiendo como tales a aquellas redes a las que se incorporan personal procedente de los ámbitos sanitarios o sociales. Finalmente y en cuarto lugar aquellas redes a las que podemos denominar normalizadas. Son aquellas que no presentan modificaciones como consecuencia de la enfermedad. Las posibles modificaciones son propias de la dinámica natural de cualquier red como motivo de cambios laborales por ejemplo. Pasemos a analizar los cuatro casos de estudio seleccionados.

\section{El caso de Antonio}

Antonio (nombre ficticio) es un varón cuya edad está comprendida entre 41 y 50 años. Reside en el área de influencia del Hospital General, soltero y en la actualidad laboralmente activo. Cuida a su madre enferma de Alzheimer. Durante el día lleva a su madre en un Centro de Día de la población de residencia. Presenta un test de Zarit de 30 puntos y en consecuencia "no existe sobrecarga del cuidador".

Su red está formada mayoritariamente por mujeres (65\%) como consecuencia de su actividad laboral. Su red está formada por tres grupos: (a) la familia por descendencia - color azul - y corresponde al 39\% del total de los miembros de su red (b) los amigos y conocidos de su infancia - color rosado - y que alcanza el $47 \%$ de su red y finalmente (c) los vecinos color gris y que alcanza sobre el total el $13 \%$.

La mayoría de tales alteri residen en la misma población de Antonio (82\%). Así mismo al $52 \%$ de los miembros de su red les pediría apoyo afectivo y al $47 \%$ les pediría apoyo material. Sus dos hermanas presentan el degree más alto, siendo la familia quien proporciona mayor apoyo afectivo.

La red de Antonio en conclusión es una red heterófila, en la que se observa la relación tanto en la esfera pública como privada; en la que no se observan cambios como consecuencia de la enfermedad del familiar atendido. Por tanto podemos señalar que corresponde a una red de tipo normalizada. 


\section{GRAFO Working Papers}

Número 4, Septiembre 2015. No 04/02. ISSN: 2014-5993

http://dx.doi.org/10.5565/rev/grafowp.15

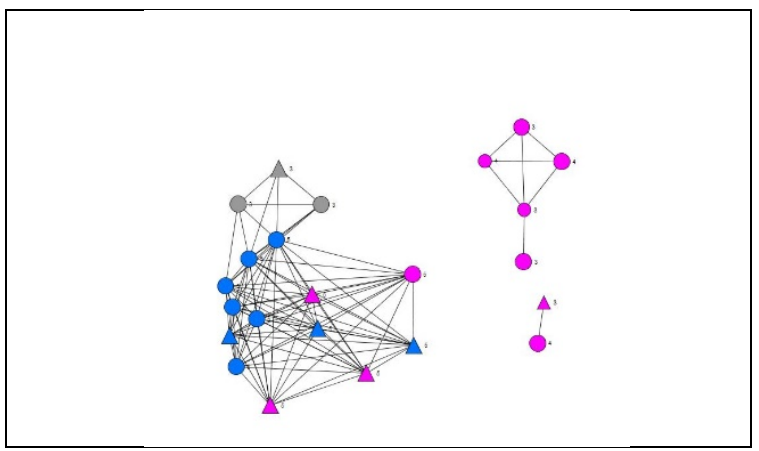

Imagen I: Red personal de Antonio (103).

\section{El caso de Elena}

Elena (nombre ficticio) tiene una edad comprendida entre 51 y 60 años. Reside en el área de influencia del Hospital y está al cuidado de su madre. Presenta un test de Zarit de 56 puntos y en consecuencia "existe sobrecarga intensa del cuidador".

Su red personal está formada exclusivamente por 11 personas distribuidas de la siguiente manera: esposo, 2 hijos, 2 vecinos, 4 amigas, 1 amistad de infancia y la madre de su pareja. En consecuencia la red personal de Elena se centra en la familia y los vecinos. El lugar de residencia de los miembros de su red corresponde en su mayoría en la misma población de Elena. Al $45 \%$ de los alteri de su red, les pediría apoyo afectivo, mientras que al $27 \%$ les pediría apoyo material.

En el caso de Elena podemos observar una red de tipo reducido. Esta circunstancia se debe probablemente a la relación existente entre el esfuerzo al que se ve sometido en el día a día por el/la cuidador/a y la relación restringida al espacio público. 


\section{GRAFO Working Papers}

Número 4, Septiembre 2015. No 04/02. ISSN: 2014-5993

http://dx.doi.org/10.5565/rev/grafowp.15

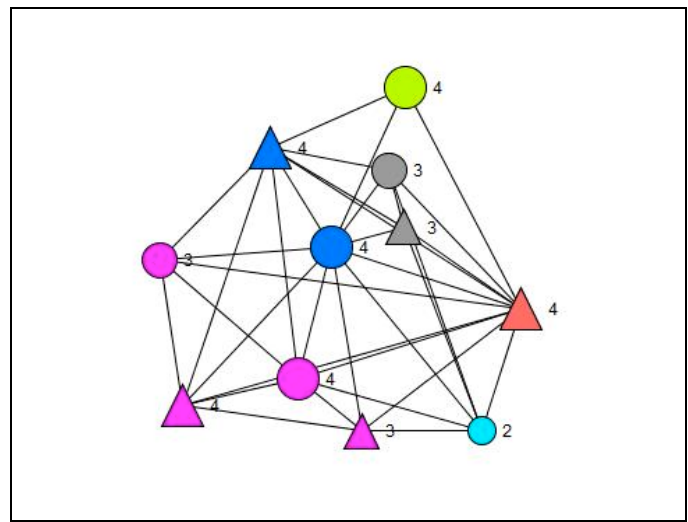

Imagen II. Red personal de Elena (207).

\section{El caso de Adela}

Adela (Nombre ficticio) es una mujer cuya edad está comprendida entre 81 y 90 años. Jubilada y al cuidado de su esposo cuya edad es similar, el cual padece una demencia importante - Cuerpos de Levy - Presenta un test de Zarit de 48 puntos y en consecuencia estamos frente a una "situación intermedia de carga del cuidador".

En el trascurso de la entrevista Adela afirma que "apenas sale de casa ya que su esposo en su ausencia, presenta cambios importantes de actitud".

Su red personal está formada mayoritariamente por mujeres (56 \%). Desde el punto de vista estructural se observa un solo grupo: la familia por descendencia/matrimonio (color azul) alcanzando el $86 \%$ del total de los alteri de dicha red. Además cita a una vecina (color gris) y a una cuidadora profesional (color verde).

La mayoría de los alteri de su red residen en la misma población de Adela (60\%). Al $47 \%$ les pediría apoyo afectivo, mientras que al $30 \%$ les solicitaría apoyo material. La centralidad de su red - degree - está ocupada por su cuñada.

Estamos frente a una red centrada exclusivamente por la familia a la que se ha incorporado una cuidadora profesional y en consecuencia estamos frente una red medicalizada 


\section{GRAFO Working Papers}

Número 4, Septiembre 2015. No 04/02. ISSN: 2014-5993

http://dx.doi.org/10.5565/rev/grafowp. 15

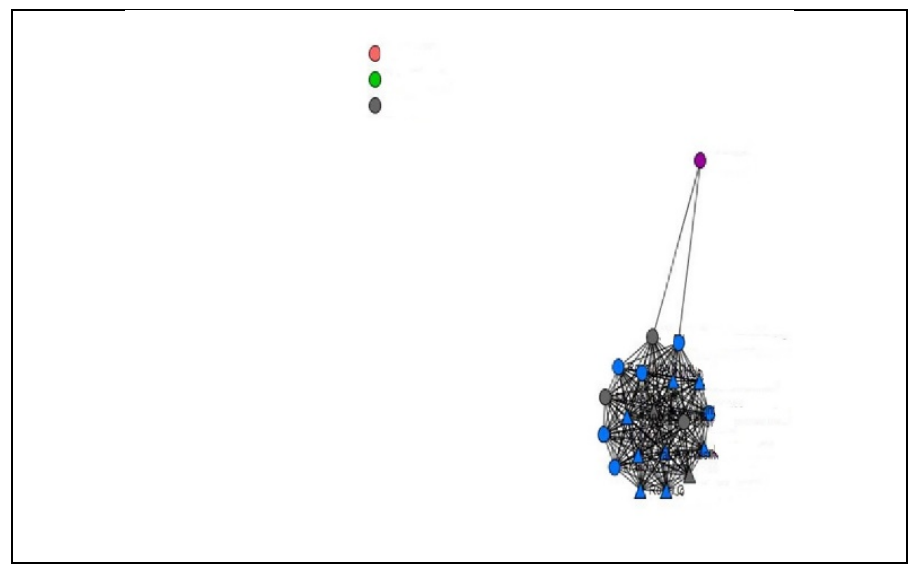

Imagen III. Red personal de Adela (401)

\section{El caso de Isabel}

Isabel (Nombre ficticio) es una mujer cuya edad está comprendida entre 41 y 50 años, que residen en el área de influencia del Parc Sanitari de Sant Joan de Déu. Está al cuidado de su madre al que se le diagnosticó la Enfermedad de Alzheimer hace aproximadamente seis años. Presenta un test de Zarit de 51 puntos y en consecuencia existe "sobrecarga moderada del cuidador".

Su red personal está formada en su mayoría por mujeres (73\%). Estructuralmente se observan dos grupos. En primer lugar los amigos que ocupan el $69 \%$ de la red - color rosa, mientras que la familia que ocupa el $21 \%$ - color azul - Destaca la presencia de una mujer que ha conocido a través del Centro de Día donde acude su madre - color negro.

EI $65 \%$ de los alteri de la red de Isabel residen en la misma población de nuestra informante. Al $47 \%$ de los alteri les solicita apoyo afectivo, al $26 \%$ les pediría apoyo material y al $21 \%$ les pediría apoyo informacional.

En consecuencia estamos frente a una red personal de tipo institucional, además de estar centrada en los amigos y la familia. 


\section{GRAFO Working Papers}

Número 4, Septiembre 2015. No 04/02. ISSN: 2014-5993

http://dx.doi.org/10.5565/rev/grafowp.15

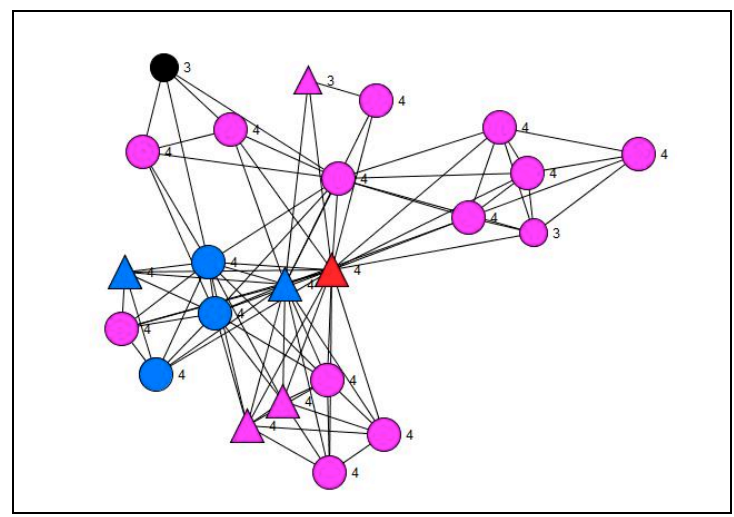

Imagen IV. Red personal de Isabel (206).

\section{Perfil del cuidador}

A través de los datos obtenidos procedentes del análisis de las RP de las personas participantes en nuestra investigación obtenemos el siguiente perfil de las personas cuidadoras de enfermos con la enfermedad que nos ocupa.

Mayoritariamente quienes atienden a una persona con EA son mujeres $(69,9 \%)$, cuya edad está comprendida entre 41 y 70 años. Son personas casadas o con pareja $(78 \%)$. En el caso de cuidadoras de sus parejas, mayoritariamente son mujeres jubiladas $(52,2 \%)$. En nuestra investigación el $65,5 \%$ poseen Estudios Primarios.

En la mayoría de los casos $(82,6 \%)$ ofrecen de forma continuada los cuidados a la persona enferma, siendo únicos cuidadores de tal enfermo $(52,2 \%)$. En aquellos casos en que existe un cuidador secundario, éste generalmente corresponde a un familiar por descendencia $(21,7 \%)$. Finalmente en la mayoría de los casos las/los cuidadoras/es que han participado en nuestra investigación no se relacionan con otros cuidadores (87\%).

\section{Quién atiende al cuidador/a}

A través del estudio de las RP de las 23 personas participantes se han obtenido un total de 480 alteri (recordemos personas citadas por ego). El 56,9\% corresponden a mujeres. Al mismo tiempo el $30,4 \%$ del total de tales alteri corresponden a familiares por descendencia, un $10,6 \%$ son familiares por matrimonio y el $28 \%$ corresponden a los amigos. Así mismo el $52,3 \%$ de tales alteri residen en el mismo barrio o población que nuestros participantes. 
Número 4, Septiembre 2015. No 04/02. ISSN: 2014-5993

http://dx.doi.org/10.5565/rev/grafowp. 15

Por otra parte el $65,6 \%$ del total de los alteri ofrecen apoyo afectivo a nuestros/as cuidadores/as, mientras que el $17,3 \%$ ofrecen apoyo material. El apoyo informacional y espiritual presentan porcentajes poco significativos.

\section{Conclusiones}

Una vez analizados los datos, las conclusiones son las siguientes:

1. Las cuidadoras y los cuidadores de enfermos con la Enfermedad de Alzheimer experimentan durante la evolución de la enfermedad de su familiar, las distintas etapas del Proceso de Duelo - en términos de Kübler-Ross. Por este motivo son frecuentes las fases de ira, sentimientos enfrentados, sufrimiento, sentimiento de culpabilidad, entre otras. Por dicha causa es fundamental la participación de profesionales y la relación entre familiares de afectados por la misma enfermedad.

2. El aumento de las cargas de trabajo como consecuencia de la cronificación de la EA, genera cambios importantes en el día a día como es la adecuación de la vivienda comportando una pérdida de la intimidad tanto del cuidador/a principal como de su pareja.

3. El perfil de la persona cuidadora de un enfermo con EA corresponde mayoritariamente a una mujer cuya edad está comprendida entre 41 y 70 años, casada, en situación de desempleo o no trabajan. Estas son además cuidadoras principales de la persona enferma, dedicando sus cuidados de forma continuada y sin remuneración alguna. Los hombres generalmente adoptan un papel secundario en los cuidados de su familiar y utilizan servicios profesionales externos. De esta manera coincidimos con los resultados obtenidos por Isla, Aldana y Guarino, entre otros. Igualmente coincidimos con los estudios realizados por Úbeda, Roca y Molina, entre otros; donde se evidencia que los cuidados de una persona enferma recaen fundamentalmente en los miembros de la propia familia.

4. La mayoría de las redes de apoyo están constituidas por mujeres procedentes del entorno más inmediato de la cuidadora o del cuidador principal (la propia pareja, los hijos, la familia y los amigos. Son precisamente estos quienes proporcionan apoyo afectivo y material a tales cuidadoras/es. Los vecinos por su parte ofrecen apoyo material.

5. La situación estresante generada por la enfermedad provoca modificaciones en las redes personales de las/los cuidadoras/es con el riesgo de la pérdidas de sus relaciones personales y consiguiente aislamiento social. En consecuencia, se pone de manifiesto la 
Número 4, Septiembre 2015. No 04/02. ISSN: 2014-5993

http://dx.doi.org/10.5565/rev/grafowp.15

importancia que adquieren en las últimas décadas del estudio de las redes sociales en el ámbito de la salud.

6. Cuanto mayor es la sobrecarga que padece la cuidadora o el cuidador, menor es su red personal. Además dicha red se centra en la familia. Por consiguiente nos encontramos frente a una población oculta en riesgo de aislamiento social.

7. Independientemente al tipo de enfermedad que padece una familiar, un/a cuidador/a adopta modelos similares de redes personales. Tales tipologías no son excluyentes entre sí y las describimos a continuación:

a. "Red reducida" son aquellas redes en las que se observa un número bajo de personas relacionadas con la/el cuidadora/r como consecuencia de la dedicación continuada y centrada básicamente en el núcleo familiar más cercano.

b. "Red institucionalizada" entendiendo como tal aquellas redes en las que se incorpora una persona conocida a través de una Institución u organización. También pueden incluirse a aquellas personas procedentes de ámbitos distintos que ofrecen apoyo: ocio, centro de culto, etc.

c. "Red medicalizada" es aquella a la que se incorporan profesionales del ámbito de la salud o social y a los que se les solicita generalmente información.

d. "Red normalizada" entendiéndose como tal aquella que no presenta cambios como consecuencia de la enfermedad de su familia. Las posibles modificaciones se producen como proceso natural en sus cuidadores: cambio de estado, cambios en el domicilio, etc. Posiblemente se trata de un estadio previo a una de las otras redes.

De esta manera coincidimos con otros estudios realizados en el ámbito de la enfermedad celíaca o las cardiopatías congénitas y llevadas a cabo por el propio autor de este artículo.

8. Nuestra investigación aporta una visión actual de la situación por la que atraviesan los/las cuidadores/as de enfermos EA. El conocimiento de las estrategias que pueden adoptar tales cuidadoras/es permite reducir la exclusión social. Es por tanto importante la visión holística de la enfermedad por parte de los profesionales sanitarios y sociales, para mejorar tanto la calidad de vida de la persona enferma como de su entorno más inmediato: familia y amigos. Para ello iniciativas como la Escola de Cuidadors pone en 


\section{GRAFO Working Papers}

Número 4, Septiembre 2015. No 04/02. ISSN: 2014-5993

http://dx.doi.org/10.5565/rev/grafowp.15

evidencia la necesidad de potenciar acciones que se dirijan a fortalecer y mantener las relaciones personales de las/los cuidadoras/es, facilitando la relación con "iguales" y ofreciendo tanto información como formación respecto a la enfermedad que sufre un familiar - en este caso EA.

Finalmente podemos afirmar que se han obtenido los objetivos previstos al inicio de nuestra investigación. Respecto al objetivo principal llegamos a la conclusión que efectivamente las redes personales de las cuidadoras y de los cuidadores se ven modificadas como consecuencia del estrés que supone la enfermedad de un familiar próximo. Así mismo se han conseguido los objetivos específicos: análisis de las redes personales, el tipo de apoyo solicitado y la procedencia de dicho apoyo.

\section{Agradecimientos}

Nuestro agradecimiento a cuantas personas han hecho posible esta investigación. En primer lugar a la Orden de San Juan de Dios que concedió la beca de investigación. En segundo lugar a los distintos Departamentos del Parc Sanitari que han dado soporte a la misma: Gerencia, Dirección Médica y de Enfermería, Unidad de Investigación, Escola de Cuidadors, Unidad de Comunicación, al personal de Recepción del Hospital General. Finalmente a cuidadores y cuidadoras han participado en la investigación con una muestra más de altruismo frente a la Enfermedad de Alzheimer.

\section{Bibliografía}

Aldana, G. y Guarino, L. (2012). "Sobrecarga, afrontamiento y salud en cuidadoras de paciente con demencia tipo Alzheimer." Summa Psicológica UST, vol. 9 (1), pp. 5 - 14.

Alzheimer Disease International (2009). Informe Mundial sobre el Alzheimer. Disponible en www.alz.co.uk (visitado el 10-05-2015).

Bazo, $M^{a} T$ (1998). "El cuidado familiar en las personas ancianas con enfermedades crónicas: El caso de los pacientes con enfermedades de Alzheimer". Revista Española de Geriatría, n 33 (1), pp. $49-56$.

Callen, A (Coord.), (2014). Manual del cuidador de pacientes con demencia...Qué nos interesa saber. Esplugues de Llobregat, Ediciones San Juan de Dios - Campus Docent.

De la Cuesta, C. (2004). Cuidado familiar en condiciones crónicas. Una aproximación a la literatura. Texto Contexto Enfermería. 13 (1), pp. 137 - 146.

Farreras, P. y Rozman, C. (1985). Medicina Interna, Vol II. Barcelona: Ed. Marín. (DOI 17a edición: doi: http://dx.doi.org/10.1016/B978-84-9022-595-0.00028-4). 
Número 4, Septiembre 2015. No 04/02. ISSN: 2014-5993

http://dx.doi.org/10.5565/rev/grafowp.15

Hollstein, B. (2002). Soziale Netwerke nach der Verwitwung. Eine Rekomstruktion der Veranderungen Informeller Beziehungen, Opladen: Leske + Budrich. DOI: http://dx.doi.org/10.1007/978-3-663-01417-1.

Isla, P (2000). "El cuidado familiar. Una revisión sobre la necesidad del cuidado doméstico y sus repercusiones en la familia". Cultura de los Cuidados, IV, № 7 y 8, pp. 187 - 194.

Kübler-Ross, E. (1993). Sobre la muerte y los moribundos. Barcelona, Grijalbo.

Llopis, J. (2006). Gluten i cor: una aproximació antropológica als canvis socials i culturals experimentats per les famílies amb fills amb malalties cróniques. Ágora d’Infermeria, 10 (3): 990-993.

Llopis, J. (2011). Corazones de papel: Patrones de cambio en las familias con niños con cardiopatías congénitas. Tesis Doctoral no publicada. Dep. Antropología Social y Cultural, Facultad de Filosofía y Letras. Universitat Autónoma de Barcelona.

Maya, I.; Martínez, M.F. y García, M. (1999). "Cadenas migratorias y redes de apoyo social de las mujeres peruanas en Sevilla". Demófilo, Revista de cultura tradicional de Andalucia. (29), pp. $87-105$.

Molina, J.M.; Lañez, M.A. y Lañez, B. (2005). El Apoyo Social como modulador de la carga del cuidador de enfermos de Alzheimer. Psicología y Salud. Vol. 15 (1), pp. 33 - 43.

O.M.S. (2007). www.who.int/mediacentre/new/release/2007/pr04/es/ (Visitado el 6-05-2015).

Prieto, C.; Eimil, M.; López, C. y Llanero, M. (2011) Impacto social de la Enfermedad de Alzheimer y otras Demencias 2011. Fundación Española de Enfermedades Neurológicas FEEN. Disponible en www.feeneurologia.com (Visitado el 11.06.2012).

Tizón, J.L. (2004). Pérdida, pena, duelo. Vivencias, investigación y asistencia. Barcelona, Paidós.

Toribio, M.E., Medrano, V., Moltó, J.M. y Beltrán, I. (2012). Red de cuidadores informales de los pacientes con demencia en la provincia de Alicante, descripción de sus características. Neurología. DOI: http://dx.doi.org/10.1016/j.nrl.2012.03.010.

Úbeda, I. y Roca, M. (2008) "Los cuidados familiares y las políticas públicas. Nursing, Vol. 26 (9), pp. 56 - 59. DOI: http://dx.doi.org/10.1016/S0212-5382(08)70805-7.

Zarit, SH.; Reever, Ke. y Bach-Peterson, J. (1980). Relatives of the impaired elderly: correlates of feelings of burden. Gerontologist, 20: $649-655$. DOI: http://dx.doi.org/10.1093/geront/20.6.649.

Zúñiga, $M^{a}$. (2007). Salud mental y apoyo social después de la ruptura conyugal: el caso de mujeres neolandesas. En Redes Sociales y Salud Pública, México (135-158). Universidad Autónoma de Nuevo León. 